\title{
Distilling the Mechanism for the Madden-Julian Oscillation into a Simple Translating Structure
}

\author{
Geoffrey K Vallis \\ Department of Mathematics, University of Exeter, \\ Exeter, EX4 4QF , UK \\ g.vallis@exeter.ac.uk
}

February 2, 2021

\begin{abstract}
This paper presents a minimal model of the Madden-Julian Oscillation (MJO), isolating a robust mechanism that leads to the observed characteristic pattern and eastward propagation. A localized heat source due to condensation at the equator leads to a Gill-like pattern in the geopotential, which in turn induces moisture convergence and further condensation. Over a wide range of parameters the moisture convergence is found to be slightly to the east of the heat source. This convergence leads to condensation and hence a heat source that also is east of the original one, thus causing the pattern itself to propagate east. The speed of the ensuing eastward propagation is limited by the ability of the moisture convergence to remain east of the moving condensation heat source. If the pattern moves too quickly, the moisture convergence cannot keep up; the propagation then slows and/or the pattern itself may dissolve. The speed of propagation thus scales with the fluid speed that is induced by the condensation itself, and thus in turn with the strength of the condensational heating, and not with a gravity wave speed. The speed also increases with the distance between the initial heating source and the subsequent condensation. In the real world this distance is determined not only by the location of moisture convergence but also by the complex physics of convection in a conditionally unstable environment, and thus cannot be accurately determined in any simple model. Thus, even though the underlying MJO mechanism is not complicated its reproduction will necessarily depend rather sensitively on model parameters in numerical simulations.
\end{abstract}

This is a non-peer reviewed article submitted to the EarthArxiv. 


\section{INTRODUCTION}

The Madden-Julian Oscillation (MJO) is, as is well known, a large-scale precipitating disturbance centered near the equator, extending meridionally to about $20^{\circ}$ North and South and with an influence in the geopotential and velocity fields that extends many thousands of kilometers. The disturbance typically forms over the warm waters of the Indian ocean and propagates eastward with a speed of around $5 \mathrm{~m} \mathrm{~s}^{-1}$, passing over the Maritime Continent and finally decaying over the cooler waters of the eastern equatorial Pacific. The phenomena may reform some tens of days later and the pattern repeats - hence it has become known as an oscillation, although in many respects it resembles a translating disturbance. The phenomenon was described by Madden \& Julian (1971) and reviews and discussions of various observations and processes have been given by Zhang (2005); Lau \& Waliser (2012); DeMott et al. (2015) and others.

In addition to analyses of the observations, attempts to understand the MJO have largely proceeded along two tracks. One is via the use of three-dimensional models, either General Circulation Models (GCMs) with parameterized convection or, more recently, near-Cloud Resolving Models (CRMs) that attempt to resolve or at least represent convection without parameterization (e.g., Liu et al., 2009; Nasuno et al., 2009; Holloway et al., 2013; Arnold \& Randall, 2015; Khairoutdinov \& Emanuel, 2018). However, many comprehensive models still have trouble reproducing the phenomenon. In the second, more theoretical, track, much simpler models of equatorial dynamics are employed, often centered around the shallow-water equations or variations thereof, with some treatment of moisture (e.g., Yano et al., 1995; Raymond \& Fuchs, 2009; Sobel \& Maloney, 2013; Fuchs \& Raymond, 2017; Vallis \& Penn, 2020). It is the second track that we follow here, but we are much influenced by the phenomenology emerging from observations and experiments with more comprehensive numerical models.

Our goal in this short paper is not to produce a complete mathematical theory of the MJO (and for reasons that will become clear we do not believe that is possible). Rather, it is to present a minimal model that distills the mechanism to a core and explains the large-scale patterns in geopotential and precipitation, providing a simple mechanism for eastward propagation and a scaling for its speed. In spite of the simplicity of explanation, the model also suggests why it is that comprehensive models often have difficulty in simulating the phenomenon.

The model draws on a previous work by Vallis \& Penn (2020), a paper that presents a more complete model with more complicated behaviour. Here we deconstruct that model and, hopefully, clarify the essential processes. The reader may wish to read that paper first, but we try to make this paper self-contained, necessitating a small amount of duplication in Sections 2 and 3. In the following section we summarize the essential phenomenology of the MJO. We then describe the mechanism we are putting forward, and describe various numerical experiments illustrating it. We then describe its relation to observations and various other models, and finally in Section 7 we give some summary remarks. 


\section{Main Features}

Observations, re-analyses, and numerical experiments with comprehensive numerical models reveal several robust features of the structure of the MJO.

1. The defining feature is a precipitation pattern centred around the equator, propagating east at a few meters per second, although the speed varies somewhat from event to event (Madden \& Julian, 1971, 1972).

2. The horizontal structure in the pressure or geopotential field resembles, after some filtering or composite-averaging, a Matsuno-Gill pattern, albeit with variations in shape and extent depending on the details of analysis (Kiladis et al., 2005; Adames \& Wallace, 2014, and others).

3. The vertical structure is relatively simple and can largely be captured by the first baroclinic mode along with a barotropic mode (Sperber, 2003; Kiladis et al., 2005).

4. Overall, diabatic heating in the MJO is dominated by latent heat release (Jiang et al., 2011; Adames \& Wallace, 2015), although radiative effects remain significant.

5. The moisture source for the precipitation is convergence in the lower atmosphere, not local evaporation (De Szoeke et al., 2015). Furthermore, there is an east-west asymmetry in the boundary layer moisture budget, with a low-level moistening ahead of (i.e., east of) the main convection zone (Hsu \& Li, 2012; De Szoeke \& Maloney, 2020). The humidity and precipitation anomalies are also strongest slightly to the east of the centre of the pattern, albeit with some differences between reanalysis products and observations (e.g., Mapes \& Bacmeister, 2012; Adames \& Wallace, 2015).

The characteristic spectral (in time) signature that serves to differentiate the MJO from a Kelvin wave (Wheeler \& Kiladis, 1999) is also well-known feature of the MJO variability. A slew of other more detailed properties of the MJO have been identified by various investigators, some more robust than others. These include the various phases of the MJO as it traverses across the ocean (Wheeler \& Hendon, 2004), a secondary quadrupole structure in the horizontal, the influence of the seasonal cycle and intra- and inter-seasonal irregularity, the interaction with the Asian monsoon, and various associated multi-scale features (e.g., smaller scale convective events surrounding the main precipitating region). Some of these are addressed in (Vallis \& Penn, 2020), but in this paper we focus on the topics in the itemized list above.

Of the above listed characteristics, the vertical structure largely follows from the effects of moist convection and associated adjustment processes, maintaining a quasi-equilibrium with a saturated adiabatic lapse rate approximately up to the tropopause. It is this structure that suggests that we can use the shallow-water equations or some variation thereof, since those equations can, in the linear approximation, be derived via an expansion (in the vertical direction) of the variables in the primitive equations into normal modes, followed by a severe 
truncation. To keep matters straightforward in this paper we will use the notation of the shallow-water equations themselves, with an additional equation for moisture, taken to be that in the lower atmosphere.

Matsuno-Gill patterns arise when there is a source of heating at or near the equator (Matsuno, 1966; Gill, 1980), and in the Gill problem specifically there is (in the canonical symmetric case) a stationary localized heat source at the equator. The precise form of the heat source does not qualitatively affect the pattern produced but can allow an analytic solution to be found. Note, though, that the MJO is a moving pattern, so cannot be precisely a Gill pattern. The heat source in the MJO problem can be associated with the latent heat release by localized precipitation and thus the production of Gill-like patterns is not particularly mysterious; rather, the question is why and at what speed does that pattern moves east.

A model of the phenomenon that produced the eastward propagation (and other variability) was offered in Vallis \& Penn (2020) using the moist shallow water equations. Evaporation from the surface provided moisture, which was then advected around and removed when the fluid became saturated. Condensation at or near the equator produced a Gill-like pattern, with convergence drawing more moisture in to the condensation zone and so sustaining the disturbance. Over a fairly wide range of parameters the ensuing condensation occurred just east of the centre of the existing pattern, generating in turn a Gill-like pattern east of the existing one. In certain circumstances this process was self-sustaining, or excitable, producing an eastward propagating precipitating disturbance.

In this paper we further simplify that model and eliminate all variability except that associated with the moving pattern itself, and thereby identify an unambiguous mechanism for the generation of the pattern and its eastward movement. We find that a fairly generic property of Gill-like patterns is that moisture convergence is slightly east of the centre of the pattern. The convergence and ensuing condensation sustain the pattern, with the offset being large enough to move the pattern east at a few metres per second but not so large as to cause the pattern to dissolve. The speed of the ensuing propagation then scales with the fluid velocity that is produced by the condensational heating, not with a gravity wave speed. Let us begin by describing the equations of motion, and then the phenomenology of the model.

\section{The Moist Shallow Water Equations}

The moist shallow water equations are, in dimensional notation and without any dissipative terms,

$$
\begin{gathered}
\frac{\partial \boldsymbol{u}}{\partial t}+\boldsymbol{f} \times \boldsymbol{u}=-g \nabla h, \\
\frac{\partial h}{\partial t}+H \nabla \cdot \boldsymbol{u}=Q_{R}+Q_{L} \\
\frac{\partial q}{\partial t}+\nabla \cdot(q \boldsymbol{u})=E-C,
\end{gathered}
$$


Here, $q$ is a specific humidity, $E$ represents evaporation, $C$ represents condensation, $Q_{R}$ is the diabatic heating due to radiative effects and $Q_{L}$ represents the latent heat release due to condensation, and other notation is standard for the shallow water equations. The momentum and height equations have been linearized about a state of rest so that $H$ is the depth of the basic state and $h$ is the deviation from this. The release of latent heat $Q_{L}$ may be related to condensation by $Q_{L}=\gamma C$ where $\gamma$ is a latent heat of condensation, although later we will choose a different recipe. The radiative damping, $Q_{R}$, is taken as a relaxation back to a flat height field. Condensation is assumed to occur on saturation, removing the excess moisture from the system almost immediately and bringing the moisture back to the saturation level. This may be represented by

$$
C= \begin{cases}\left(q-q_{s}\right) / \tau & q>q_{s} \\ 0 & q \leq q_{s}\end{cases}
$$

where the timescale $\tau$ is small compared to other timescales in the system, and $q_{s}$ is the saturation specific humidity which we take to be an exponential function of the height field, varying as

$$
q_{s}=q_{0} \exp (-\alpha h)
$$

where $\alpha$ is a constant.

Evaporation from the surface is represented as

$$
E= \begin{cases}E_{q}\left(q_{g}-q\right) & q_{g}>q \\ 0 & q_{g} \leq q\end{cases}
$$

where $E_{q}$ and $q_{g}$ are constants and the conditional statement in (4) prevents dew formation. See Vallis \& Penn (2020) for a more detailed description of all these terms. In the rest of the paper we nondimensionalize lengths by the equatorial deformation radius $(c / 2 \beta)^{1 / 2}$, time by the factor $(2 c \beta)^{-1 / 2}$ and velocities by $c$, where $c=\sqrt{g h}$. We also use the geopotential $\phi=g h$, which is proportional to pressure. In the above, the parameterization of precipitation and its latent heat release is particularly uncertain, and that uncertainty remains in comprehensive three-dimensional models.

\section{Humidity in the Gill Solution}

\subsection{Moisture convergence in the Gill solution}

Let us first suppose that the velocity and geopotential fields are given by the canonical Gill solution itself, the analytic expressions for which are given in the Appendix. Suppose also 


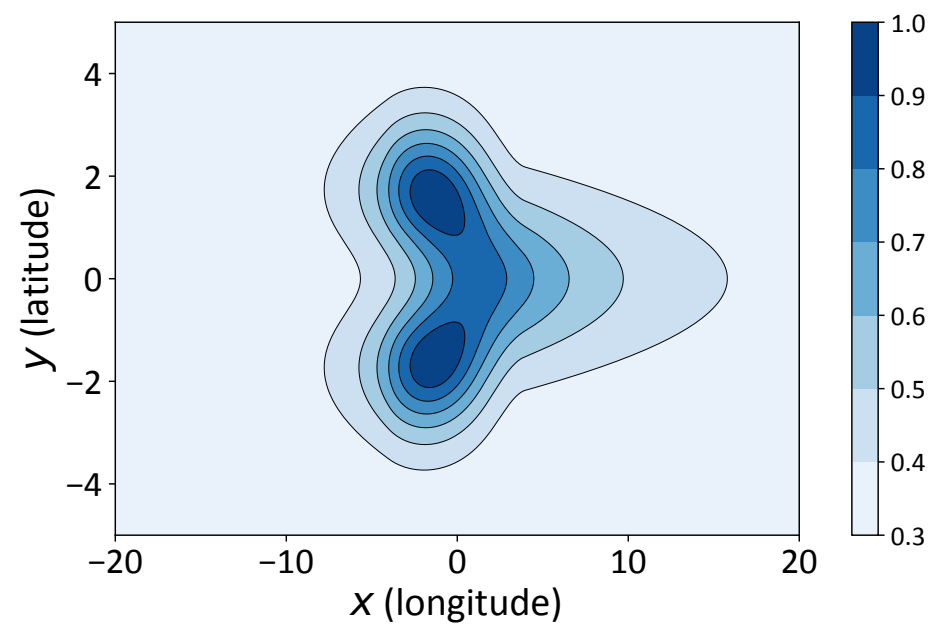

Figure 1 Saturated moisture field, $q_{s}$, as given by (5), scaled by its maximum value, associated with the Gill solution with a heat source centred at $(0,0)$. The horizontal axes (here and in subsequent figures) are scaled by the equatorial deformation radius.

that the atmosphere has a constant relative humidity (which can be taken to be unity) so that

$$
q=q_{0} \exp \left(-\alpha \phi_{G}\right)
$$

where $\phi_{G}$ is given by (9) and we rescale $\alpha$ from (3). This specific humidity field, shown in Fig. 1 has a similar structure to the Gill pattern itself. However, the humidity convergence differs slightly from the velocity convergence as illustrated in Fig. 2 and Fig. 3: specifically, the center of moisture convergence is slightly to the east of the heating source that provides the pattern in the first instance, and in this case the distance is about one equatorial deformation radius. This result is dependent on the saturation value of the moisture being a function of geopotential (and thence temperature); if it were constant the moisture convergence at saturation would be just the same as the velocity convergence, with a maximum virtually coincident with the centre of the heating itself.

\subsubsection{Evolving the moisture}

A similar result regarding the humidity convergence holds if we numerically integrate the moist shallow water equations, i.e. equation set (1) to (4), forward in time. In the first instance we force the equations with stationary localized heat source at the equator independent of the moisture field, and treat moisture as a passive tracer. The pressure and velocity fields then evolve into a familiar Gill-like pattern. The moisture now enters the system by evaporation, is advected around and condenses upon saturation, and the convergence of velocity and moisture in a typical solution are shown in Fig. 4. The convergence now forms a narrow region east of the heating, as does the condensation (i.e., the region of saturation) and in this case the condensation and moisture convergence are almost coincidental.

The nature of this result is not sensitive to the parameters chosen to represent evaporation (in equation 4), nor to the particular form of heating used to produce the pattern. In Fig. 4 a heating pattern similar to that of the original Gill problem was used. If we use either a very 

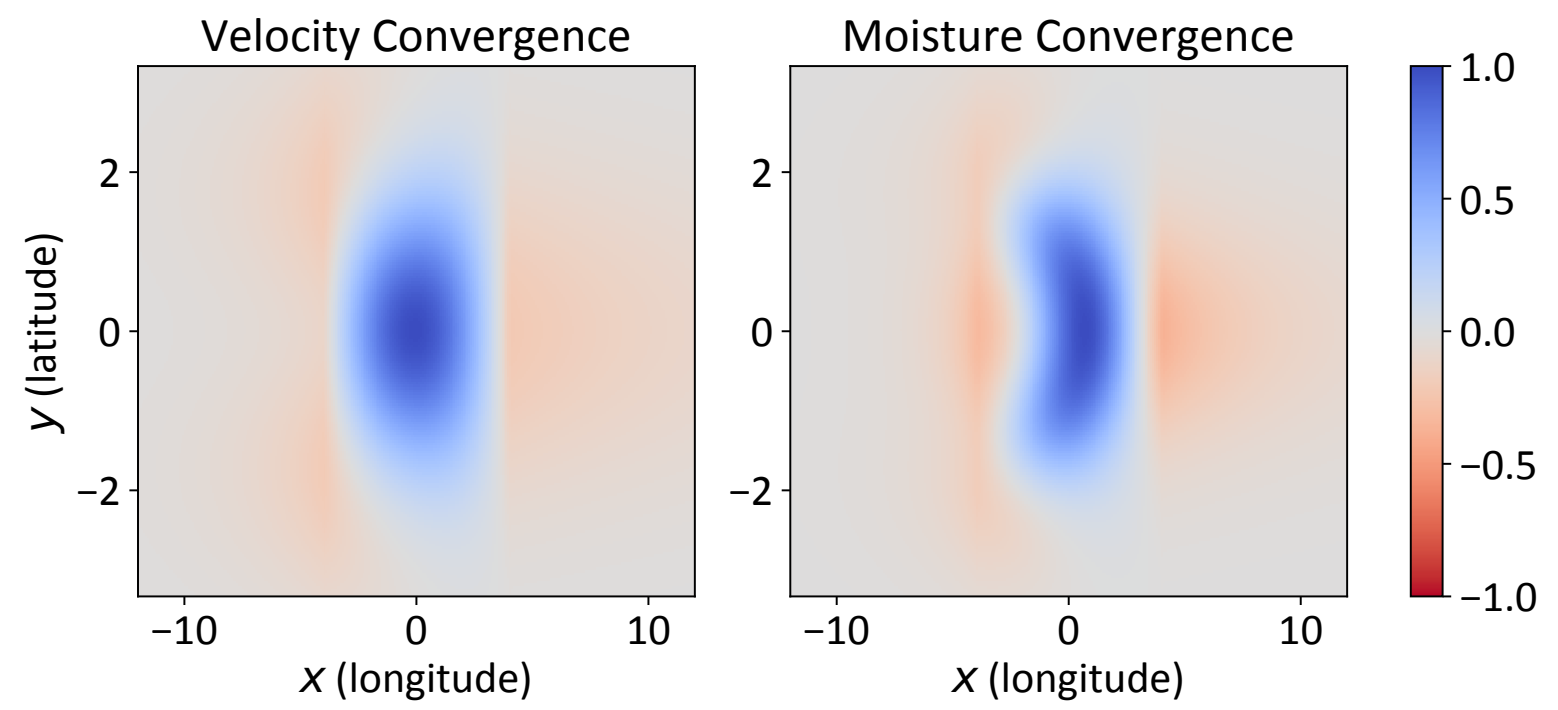

Figure 2 Convergence of velocity, $-\nabla \cdot \boldsymbol{u}$, and the saturated value of moisture $-\nabla \cdot(\boldsymbol{u} q)$ in the Gill solution. Both fields are normalized by their maximum value, and the horizontal axes are scaled by the equatorial deformation radius. The velocity convergence is centred on the point $(0,0)$ whereas the moisture convergence is east of that, as seen clearly in Fig. 3.

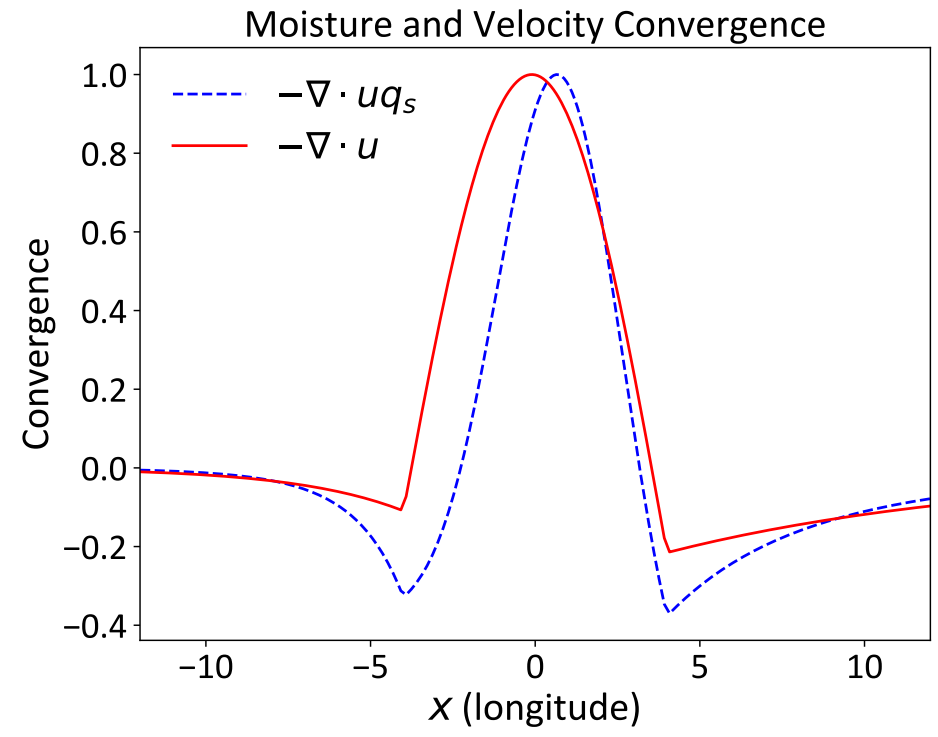

Figure 3 As for Fig. 2, but now showing convergence of velocity, $-\nabla \cdot \boldsymbol{u}$ (red curve), and the saturated specific humidity, $-\nabla \cdot\left(\boldsymbol{u} q_{s}\right)$ (blue dotted curve) at the equator. Both fields are normalized by their maximum value.

meridionally extended diabatic heating pattern, extended several deformation radii north and south, or a zonally extended pattern, a similar result emerges. We explore this further later on, and the reader may look ahead to Fig. 7.

\section{Eastward Propagation}

Now suppose that, instead of being imposed, the heat source producing the pressure pattern is that produced by the condensation itself. Then, since the heating is east of the original source the system will be pulled toward the new source by the pressure field, shifting the condensation 

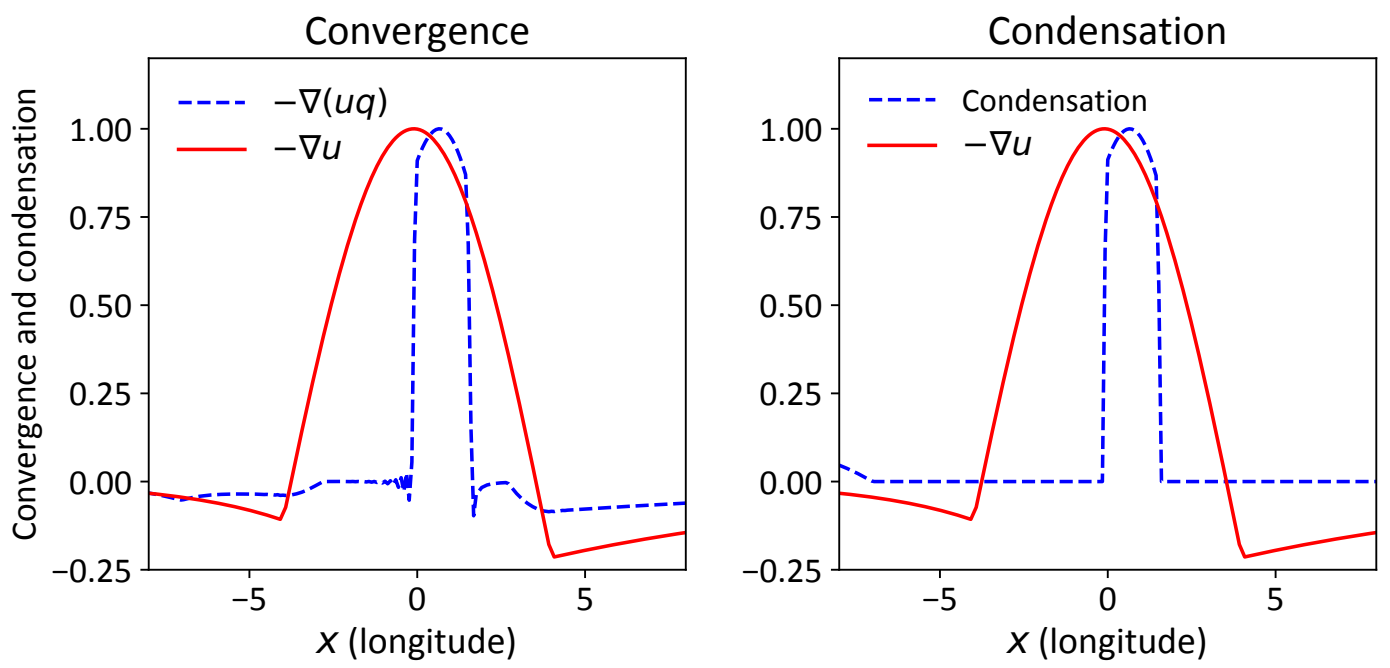

Figure 4 The convergence of moisture (left panel, blue dashed line) and the condensation (right panel, blue dashed line), along with the velocity convergence (red solid line) in both panels, in a numerical solution of the moist shallow water equations with a specified, stationary, heat source centred at $x=0$. All fields are normalized by their maximum values.

eastward and giving rise to a continuous eastward propagation. Before showing this result in the shallow water equations we illustrate the process with a simple but informative toy model.

\subsection{A very simple model}

Suppose $X_{P}$ is the position of the centre of the pattern of the pressure field and that $X_{C}$ is the position of the condensation, and that the condensation then provides a heat source toward which the pattern is drawn. If $X_{P}$ relaxes towards $X_{C}$ at a rate $k_{1}$ we may write

$$
\frac{\mathrm{d} X_{P}}{\mathrm{~d} t}=k_{1}\left(X_{C}-X_{P}\right)
$$

If the pressure pattern at a given time is such that the moisture convergence and condensation are a distance $\Delta X$ east of the centre of the pressure pattern, and the centre of the condensation is drawn toward that position at a rate $k_{2}$, then the position of the condensation will evolve according to

$$
\frac{\mathrm{d} X_{C}}{\mathrm{~d} t}=k_{2}\left(\left[X_{P}+\Delta X\right]-X_{C}\right) .
$$

The solution of the above two equations, after the initial transient has died, is

$$
X_{P}=X_{0}+\frac{k_{1} k_{2} \Delta X}{k_{1}+k_{2}} t
$$

where $X_{0}$ is a constant, and similarly for $X_{C}$. Two limiting cases are particularly informative. If $k_{2} \gg k_{1}$ then $X_{C} \approx X_{P}+\Delta X$ and (6a) becomes $\mathrm{d} X_{P} / \mathrm{d} t=k_{1} \Delta x$, and the entire system propagates east at a speed $k_{1} \Delta X$. If $k_{2} \ll k_{1}$ then $X_{C} \approx X_{P}$ and (6b) becomes $\mathrm{d} X_{C} / \mathrm{d} t=k_{2} \Delta X$, 

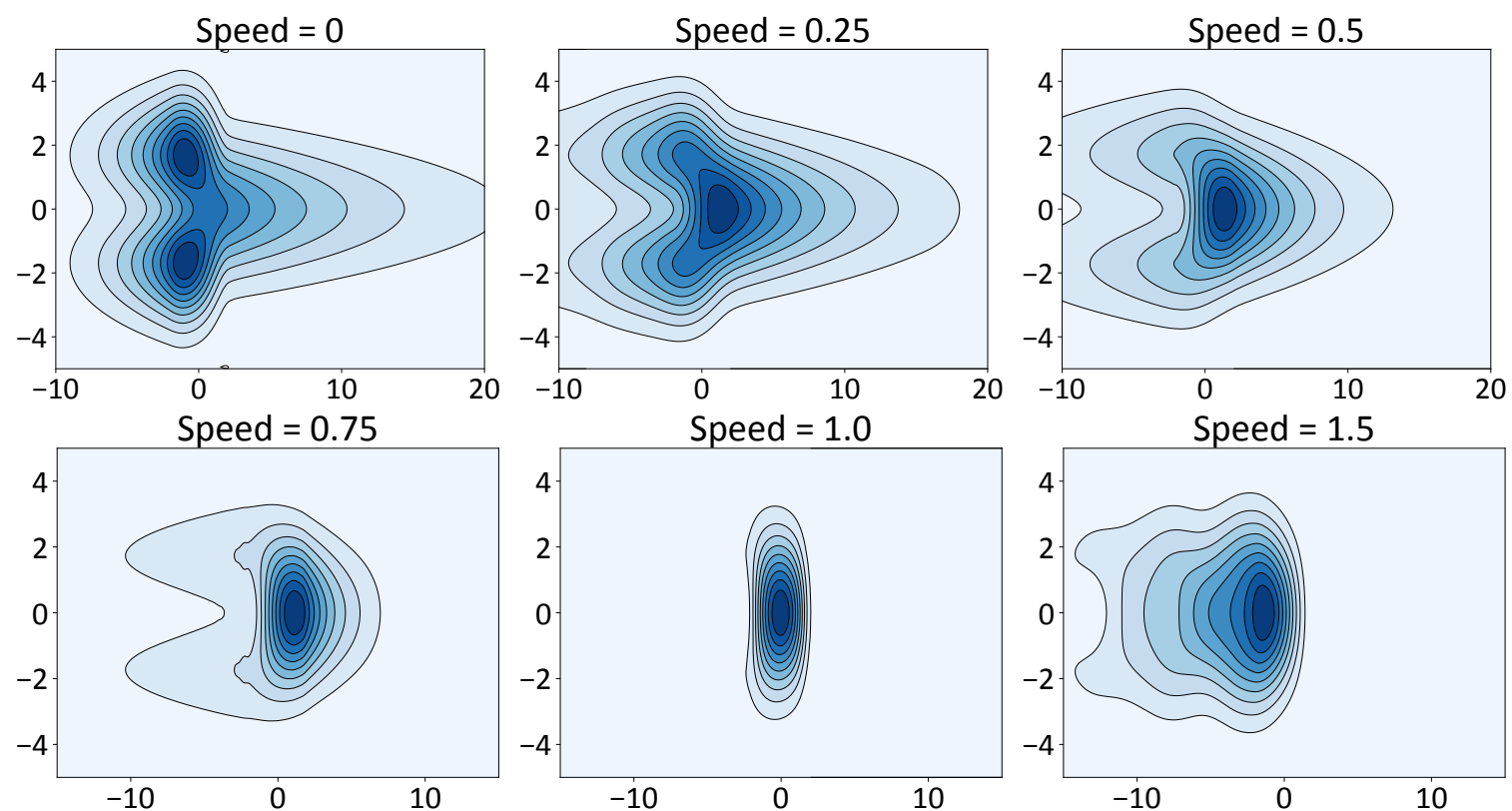

Figure 5 Pressure field in solutions of the shallow water equations with a moving heat source. The $x$ and $y$ co-ordinates are scaled by the deformation radius and the speed is scaled by the gravity-wave speed. The heat source is localized at the equator, with a horizontal scale of about two deformation radii, moves eastward at a constant speed and for plotting purposes the $x$-axis is shifted so that the center of the heating at the time of the plots is always at $x=0$.

and the system also propagates east, now at a speed $k_{2} \Delta X$ but with the centre of the pressure now almost coincident with the precipitation. It is the second limit that is more relevant in the MJO case, since the moisture convergence forms on a slow advective timescale whereas the pressure pattern forms on a timescale determined by the faster gravity wave speed. In both cases, though, the speed is proportional to the nominal offset, $\Delta X$, between the precipitation center and the pressure center. That distance cannot be quantitatively determined by a simple model, or even the full moist shallow water equations, since it depends on details of convection and radiation. This precludes formulating an MJO theory that is both simple and quantitatively accurate, but not progress.

\subsection{Numerical integrations of the equations of motion}

We now show results arising from various numerical integrations of the shallow water equations. We do not allow a complete interaction between the moisture and the dynamics (as was done in Vallis \& Penn (2020)). Rather, we specify the diabatic heating to be at or near the center of the condensation, with a specified strength and spatial structure. We are thereby able to probe in a controlled fashion how the speed of propagation depends on the nature of the condensation and other parameters of the problem. 

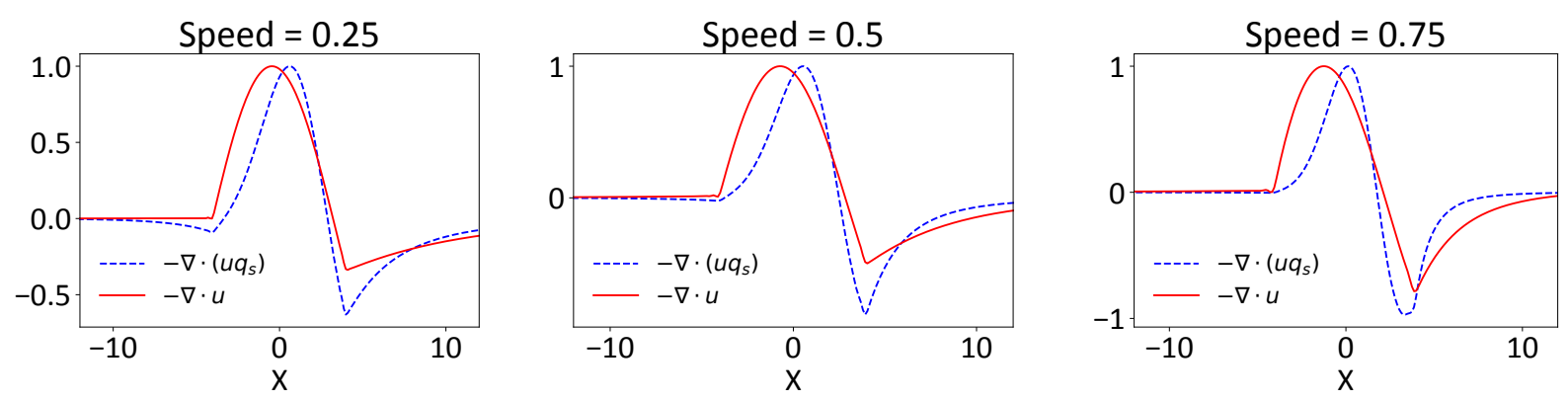

Figure 6 The velocity divergence and the humidity convergence at the equator corresponding to three of the simulations (as labelled) in Fig. 5. The humidity is the saturated value given by (3) using the height field of Fig. 5.

\subsubsection{Integration of dry equations with a localized, moving heat source}

In the first instance we integrate the dry shallow water equations (i.e. no moisture field) with a localised heating source at the equator. The heating source is specified to be that in the Gill problem except that we move it eastward at a specified speed $U$. The results (Fig. 5) show that the pattern can maintain its coherence for values of $U$ up to values of order $c$, the gravity wave speed. At speeds above $c$ the pattern in unsteady. It is the pressure field that is organizing the pattern and information is passed at a speed $c$, so the result is not surprising. Related results were obtained by Penn \& Vallis (2017) in the context of a not-quite tidally-locked planet, in which the sub-stellar point moves across the planet at a non-zero speed. The eastward movement of the source does modify the pressure pattern, in particular by shortening the leading (eastward pointing) Kelvin lobe and (in all but the resonant case when the source speed is one) lengthening the trailing Rossby lobes to something more of a swallowtail pattern, as might be expected since the source is moving in the same direction as the Kelvin waves.

For speeds up to about three-quarters of the gravity wave speed $(0.75 c)$ where a Gill-like pattern can be clearly distinguished, the convergence of the saturated value of humidity (that is, $-\nabla \cdot\left(\boldsymbol{u} q_{s}\right)$ where $q_{s}$ is calculated using ((3) and the height field of the evolving solution) remains eastward of the heat source (Fig. 6. This speed then provides an upper bound on the speed at which the MJO pattern may move. However, the gravity wave speed in Earth's tropical atmosphere is of order $20-30 \mathrm{~m} \mathrm{~s}^{-1}$ so that maintaining the coherence of a Gill-like pattern is evidently not the main determinant of the speed at which the MJO pattern moves (about $5 \mathrm{~m} \mathrm{~s}^{-1}$ ).

\subsubsection{Integrations with an interacting humidity field}

We now evolve the humidity field alongside the usual shallow water equations. However, we constrain the effects of the release of latent heat to occur only in a prescribed single region of specified strength at a position determined by centre of the moisture convergence. In this way we eliminate all but a single condensing region, allowing for the potential formation and 

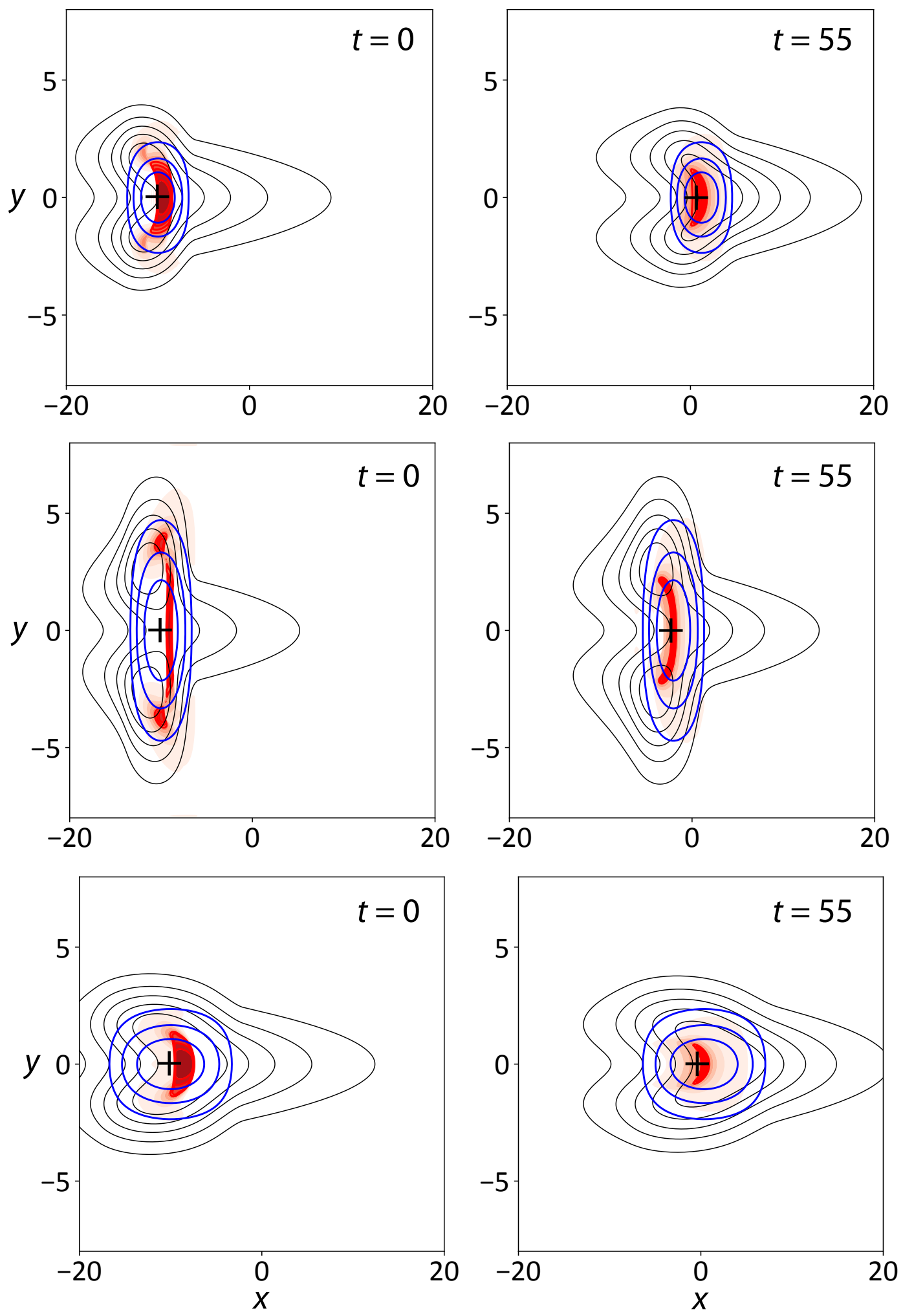

Figure 7 The pressure (black contours), the moisture convergence (red shading) and the associated heating (blue contours) at the nondimensional times shown. The cross (plus sign) indicates the centre of the velocity convergence. The left panels show the fields initially with the heat source prescribed and stationary, and the precipitation then forms just slightly east of that. The heat source is then allowed to respond to the position of the condensation, causing the pattern to translate east. 
movement of an MJO-like event but no other condensing structures.

Typical solutions are shown in Fig. 7 and Fig. 8. The equations are initially integrated for a period of time with a specified, stationary heat source centred at the equator, representing a warm pool, with moisture treated as a passive tracer. The pressure field evolves in a Gill-like pattern and the associated velocity field converges moisture, producing precipitation east of the heat source, just as in Fig. 4. Once a steady state is reached the heat source is allowed to follow the condensation; this causes the pressure pattern to shift east; the moisture convergence is thus also shifted east, causing the entire system to propagate eastward.

In the top panel of Fig. 7 the condensational heat source (blue contours) is approximately isotropic, extending a couple of deformation radii both zonally and meridionally. In the middle panel the heating is extended meridionally, whereas in the bottom panel the heating is extended zonally. The far field to the east is similar in all cases, since this is determined by the Kelvin wave response to the heat source at the equator. It is this long 'fetch' that is bringing in moisture ahead of the centre of the pattern, and eastward propagation occurs in all cases at speed of about $0.18 c$, which if $c \approx 25 \mathrm{~m} \mathrm{~s}^{-1}$ is about $4.5 \mathrm{~m} \mathrm{~s}^{-1}$. This speed is determined in part by the strength of the condensation, which is such as to produce fluid velocities that are also a few metres per second, as further explored below.

Note that initially, when the heat source is fixed independently of the moisture, the condensation is noticeably east of the heating source, just as in Fig. 4. But once the heat source is taken to be at the location of the condensation the pressure pattern can almost immediately 'catch up', because its response time, being set by the gravity wave speed, is shorter than the advective timescale of the moisture. Thus, in all of the left-hand panels of Fig. 7 condensation is noticeably east of the heat source, whereas in the right hand panel the offset is smaller. Nonetheless, it is the fact that moisture converges toward the east of center of the pressure pattern that enables the pattern to move eastward. Much of the moisture is drawn in from the warm Kelvin lobe to the east but there are also meridional fluxes around the trailing Rossby lobes. The difference between the moisture fluxes $(\boldsymbol{u} q)$ and velocity itself $(\boldsymbol{u})$ is not marked it is the variation of the velocity field that is primarily responsible for the convergence itself but is important.

\subsection{The speed of propagation}

In the simple model of Section 5.1 the speed of propagation is proportional to the displacement, $\Delta X_{C}$, and (in the relevant limit) the inverse timescale, $k_{2}$. We now explore whether and how the speed of propagation of the MJO depends on the analogous quantities in solutions of the equations of motion.

\subsubsection{The displacement}

In the MJO the equivalent displacement is the offset between the location of the center of the

pattern (where the horizontal velocity converges) and the location of the latent heat release 

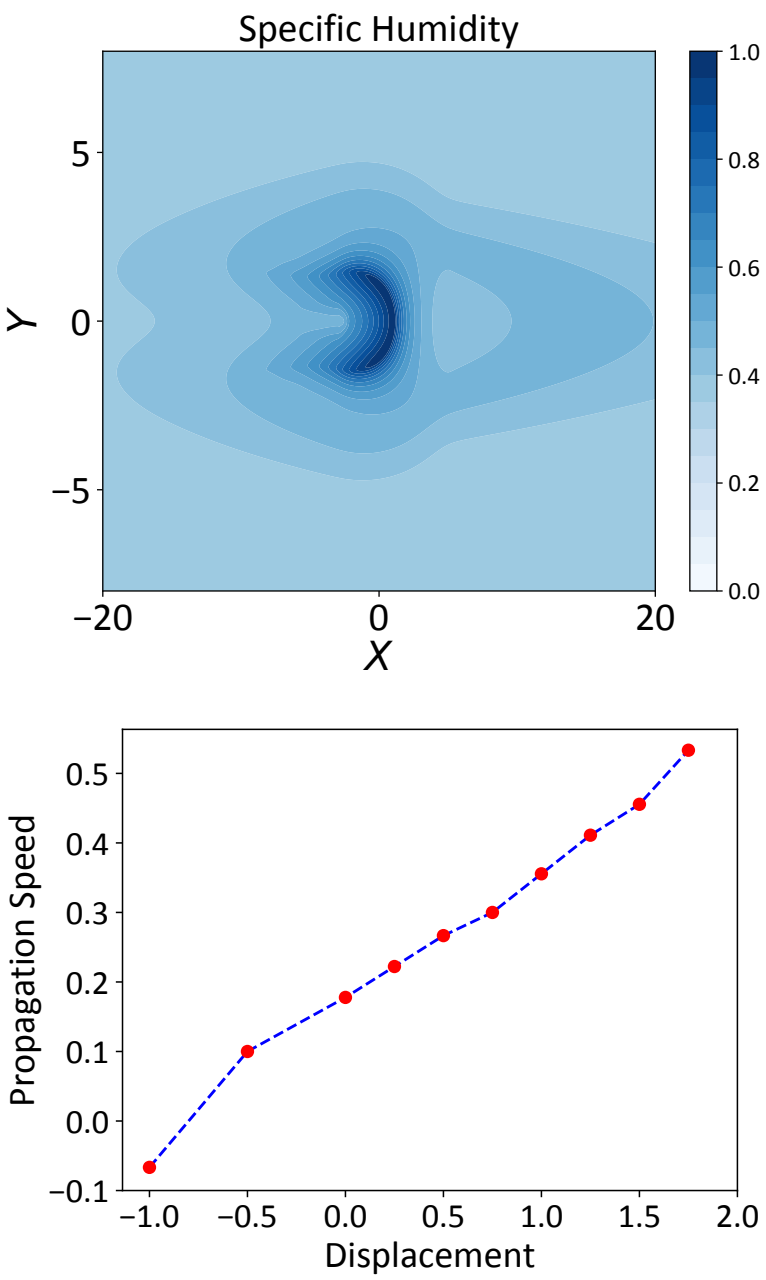

Figure 8 Snapshot of the specific humidity, scaled by its maximum values, in a numerical integration of the moist shallow water equations. The pattern is moving eastward at a speed of about $0.2 c$ or about $5 \mathrm{~m} \mathrm{~s}^{-1}$

Figure 9 Speed of propagation of the MJO pattern as a function of the specified displacement of the moisture condensation from the position of maximum moisture convergence. Velocities are scaled with the gravity wave speed, $c$ and the displacement by the deformation radius.

due to the precipitation. The latter cannot be accurately determined in any model short of a comprehensive three-dimensional model that has an accurate treatment of convection and radiation, although it will be broadly determined by the moisture convergence. Thus, rather than using a specific and perhaps ad hoc parameterization, a sequence of experiments is performed (with the moist shallow water equations) in which the center of the heat source arising from the precipitation is displaced relative to the position of the moisture convergence. The heating is centered at the equator, with a given strength and extends a few deformation radii both zonally and horizontally, as in the blue contours in the top panel of Fig. 7 . In the integrations shown in Fig. 7 the condensational heating is set to be coincident with moisutre convergence, which is about a deformation radius east of the velocity convergence; the pattern then propagates east at a few metres per second. As the specified displacement is increased Fig. 9 shows that the propagation speed increases approximately linearly, as in the model of Section 5.1. This result does not depend on the specification of the heating structure - it holds in all the patterns shown in Fig. 7. 


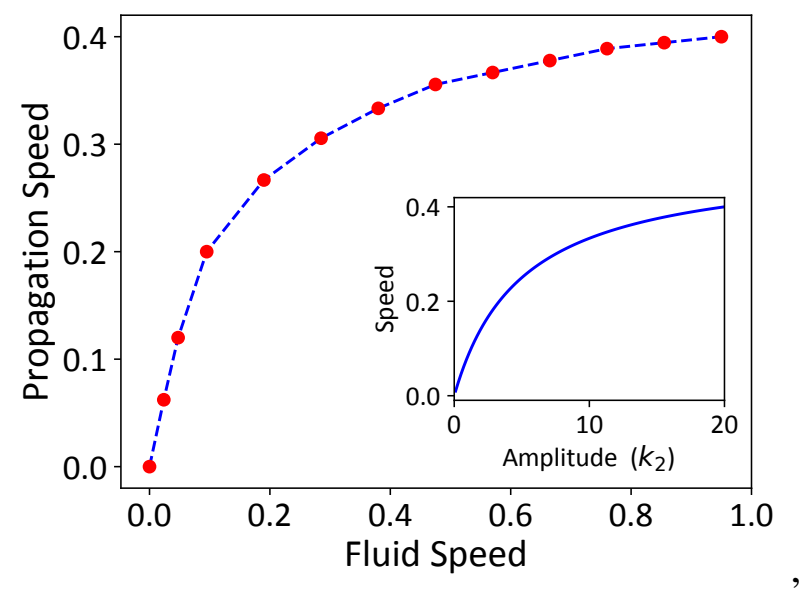

Figure 10 Speed of propagation of the MJO pattern as a function of rms fluid speed (which is directly proportional to the amplitude of the pattern). Values are nondimensional, scaled with the gravity wave speed, $c$. The inset shows a corresponding plot for the toy model with speed given by (7), where $k_{2}$ is the inverse timescale of the moisture convergence and $k_{1}=5$ and $\Delta x=0.1$.

\subsubsection{The disturbance amplitude}

The disturbance amplitude is largely determined by the strength of the condensational heating; that is, the amplitude of $Q_{L}$ in (1b)). In a sequence of experiments we vary the strength of this heating, keeping its position relative to the moisture convergence fixed. The results are shown in Fig. 10, with the average fluid velocity along the equator used to represent the disturbance amplitude. As expected the speed increases with the disturbance amplitude. The result arises because the fluid velocity determines the timescale on which the moisture, the advected variable, can respond to changes in the pressure field, and that is the limiting factor in the adjustment of the whole pattern. For small values of the amplitude the speed grows linearly with the amplitude before tapering off at large amplitude. At these large amplitudes the fluid speed is approaching that of the gravity wave speed so that the adjustment time of the pressure is no longer significantly shorter than that of the humidity. Similar behaviour is seen in the very simple model (inset to Fig. 10) when the parameter $k_{2}$ is varied. That parameter essentially represents the fluid velocity divided by a characteristic length scale of the pattern.

Evidently, at speeds lower than the gravity wave speed (which is the realistic regime) the propagation speed scales advectively, meaning it scales with the fluid speed; it is limited by the advection of moisture and is not directly related to the gravity wave speed. The translation speed is similar to or somewhat greater than a typical fluid speed associated with the disturbance (and this is also the case in the observations, as noted later). It need not be thought of as a phase speed or group velocity associated with a wave except in some rather general sense. Rather, it is the speed at which a coherent structure translates.

To determine the amplitude of the disturbance from first principles in anything short of a full numerical model is, given the complexity of precipitation processes, a Sisyphean task. In Vallis \& Penn (2020) condensational heating was specified as $Q_{L}=\gamma C$. where $C$ is the local condensation and $\gamma$ is the latent heat of condensation. This is a simple and defensible recipe but it should not necessarily be regarded as an accurate scheme. If we wished to represent the process in a simple model then, noting that the condensation rate depends on the rate at which humidity converges, which in turn depends amplitude of the disturbance itself, we might write 
an equation of the rather general form

$$
\frac{\mathrm{d} A}{\mathrm{~d} t}=\mathcal{F}(A)-\mathcal{D}(A) .
$$

where $\mathcal{F}(A)$ is the latent heat release, which is an increasing function of $A$, and $\mathcal{D}(A)$ represents dissipative processes, also an increasing function of $A$. The function $\mathcal{F}(A)$ is in reality quite complicated; it may be predominantly linear (since the moisture convergence depends approximately linearly on the disturbance amplitude) but depends on the nature of the flow field. It may also have nonlinearities that depend on $q$ and other factors. The dissipation is also, in reality, quite complicated, depending on radiative damping, surface drag and gravity wave radiation, and it may be nonlinear. The point, though, is that (8) can plainly give rise to instability and a great many process will contribute to that. The ensuing balance will determine the amplitude of the resulting disturbance (and that may be zero). Here we sidestep the difficulties of determining that balance by specifying the strength of the condensational heating.

\subsection{Moisture as the only prognostic variable}

It is possible to simply the model further (while still staying within the framework of the fluid equations of motion) by exploiting the fact that the pressure field responds very quickly to the moisture field. Thus, we may evolve the moisture field according to (4) and suppose that the pressure and velocity fields respond instantly to the diabatic heating arising from the precipitation. The moisture convergence and precipitation is then calculated just as in the solutions above, but the system now has just one prognostic equation. If the diabatic heating is given a simple form then the model may almost be specified analytically, but a numerical implementation is far more practical.

Solutions of this system (not shown) are very similar to those given above, and in particular the pattern propagates east at a similar speed to those shown. Using parameters that produce higher translation speeds the solutions begin to differ in certain quantitative aspects because the model cannot capture the difference between the structure of a stationary Gill-like solution and a moving solution. Nonetheless, the essential mechanism of pattern formation and propagation is captured by such a model.

\section{Observations and Other Models}

\subsection{Observations}

The overall patterns of the model presented above are broadly in accord with observational analyses (and re-analyses) in a number of respects, and in particular all of the listed features in Section 2 are accounted for. As many authors have pointed out, the general horizontal structure 
of the MJO has similarities with a GIll pattern. Here this is a unavoidable consequence of a localized heating centred around the equator, caused by the latent heat released by the precipitation. It is this pattern that itself causes the moisture to converge, so sustaining the pattern. The actual solution will differ from a true Gill solution because of two effects: first, the MJO solution is moving, giving it somewhat longer trailing Rossby lobes; second, the distribution of latent heat release by the moisture is not in general the same as the heating in the original Gill problem. However, neither of these have a particularly large effect, and the far field does not care about the precise nature of the heating.

Observations do show that latent heat release is the dominant diabatic heating in the MJO system (Jiang et al., 2011; Adames \& Wallace, 2015), producing anomalous velocities of a few metres per second (Adames \& Wallace, 2014). Relatedly, observations show that moisture convergence, rather than local evaporation, is the primary supply of the precipitation in an MJO event (De Szoeke et al., 2015). Evaporation is fairly constant over the warm equatorial region, whereas precipitation anomalies associated with the MJO are an order of magnitude larger than local evaporation anomalies. The moisture convergence arises both zonally (coming in from the east along the Kelvin lobe) and meridionally, around the trailing Rossby lobes, and occurs in the lower atmosphere. In all these aspects the model is broadly consistent with observations.

A more stringent comparison arises by looking at the relative phases of pressure, velocity and moisture in the pattern as it propagates east. The moisture fluxes $(\boldsymbol{u} q)$ are visually rather similar to the velocity vectors $(\boldsymbol{u})$, and the convergence is associated mainly with changes in velocity field rather than moisture itself, in both model and observations, with subtler differences accounting for the difference in their exact phase. Observations show that convergence in the lower atmosphere increases moisture levels ahead of the convection (De Szoeke \& Maloney, 2020). This offset is present in the model; in fact it is central to the eastward propagation. It was also earlier suggested by $\mathrm{Hsu} \& \mathrm{Li}$ (2012) as a possible factor in MJO propagation, and it is a key factor in GCM simulations of the MJO (Hsu et al., 2014). The notion of low-level convergence leading (i.e., being eastward of) the existing convection zone was also noted by Zhang \& McPhaden (2000); Sperber (2003) and Maloney (2009), albeit with some differences of interpretation.

The eastward shift of precipitation relative to the centre of the pattern is key to the eastward propagation and is present in observations. But it should be said that observations (TRMM and GPCP) and re-analyses are not wholly in agreement as to the extent of the shift (Mapes \& Bacmeister, 2012; Adames \& Wallace, 2015). Results from the model suggest that eastward offset may be more evident in the early stages of the MJO, when the MJO is forming and the heating is in part due to a warm sea surface. Later in the cycle the moisture convergence and the velocity convergence are more nearly coincidental, as seen by comparing the right and left columns in Fig. 7, because of the fast response of the pressure and velocity fields. Finally, we remark that the model does not seek to explain smaller scale features of the MJO, such as the convective features surrounding the precipitating region. These arise in the numerical 
simulations of Vallis \& Penn (2020), but are here regarded as inevitable consequences of the interaction of condensation and the pressure field rather than as an essential feature of the MJO itself.

\subsection{Other models}

Many previous studies have emphasized the importance of moist interactions in producing an MJO, and here we discuss some that have connections with the model presented here, notably the 'moisture mode' theories, as presented in (Raymond \& Fuchs, 2009) and (Sobel $\&$ Maloney, 2013) and other articles by those authors. Although various aspects differ, in those theories the interaction of moisture, precipitation and advection is essential, as it is here. The eastward propagation of the MJO is associated with horizontal advection by anomalous lower-tropospheric winds resulting in an increase in humidity east of the enhanced convection and a drying west of the convection. However, there are differences, both between the types of moisture-mode models themselves, and with the model here. For example, the Sobel-Maloney model is one-dimensional, makes certain specific assumptions as to the relation between precipitation and column water vapour, and radiative effects seem essential in destabilizing a column and maintaining the system. Here, we do not focus on that instability, although the specification of the release of latent heat with a given strength may implicitly account for it. In some of the Raymond-Fuchs models wind-induced evaporation was found essential in destabilizing the system and giving rise to propagation. Here, wind-induced evaporation plays no explicit role, although it was found to be a destabilizing feature in Vallis \& Penn (2020) that enhanced the eastward propagation.

The 'trio interaction model' of Wang et al. (2016) uses a variant of the moist shallow water equations and is presented as an integrative framework that includes moisture-mode and other theories. It has relatively elaborate parameterizations for physical processes - a cumulus parameterization, for example - and the authors couch their results in terms of an instability and a propagating mode. However, the resulting picture has similarities to that here, in that a Gill-like pattern emerges and moisture convergence evidently leads the convective heating. The eastward propagation is attributed to a variety of effects, including convective heating from the warm pool SST, the coupling of Kelvin and Rossby waves and the moisture feedback. In the model presented here the warm pool (i.e., a stationary heat source) is only necessary for the MJO genesis. Once begun, the system is naturally self-sustaining and moisture feedback alone is sufficient to provide propagation.

The model presented by Vallis \& Penn (2020) uses the same core equations as those

presented here. It differs only in that it allows a direct interaction with the moisture condensation and the pressure field; that is, condensation on saturation leads immediately to heating with no additional filtering or parameterization. Eastward propagation occurred when the model was 'excitable', meaning that the interaction of moisture and the dynamics led to an instability and thence to self-sustained variability. The excitability is a function of such 
things as the dissipative parameters, the release of latent heat, and the presence or otherwise of wind-enhanced evaporation. Parameters controlling radiative destabilization and cloud parameterizations would also play a role if included, potentially enhancing or suppressing the instability depending on their formulation. The model produces multi-scale variability, and preferential formation over a warm pool, both features of the observed system. However, the essential mechanism of eastward propagation is the same as that presented here - namely condensational heating occurring slightly to the east of the centre of the pattern, setting up a pressure field that pulls the pattern toward to that heating. There are two reasons there for the slight eastward offset: (i) Moisture convergence itself is slightly eastward of the centre of the pattern, because the warm moist air is drawn in along the leading Kelvin lobe (as here), and (ii) Kelvin waves emitted by the convection propagate eastward, so triggering more condensation east of the pattern centre.

\section{Conclusions}

This paper has presented a model that gives rise to the structure of the MJO and exposes the mechanism for its eastward propagation in a simple way, without attempting to be complete. The broad features of the three-dimensional structure of the MJO follow straightforwardly if latent heat release is the dominant form of anomalous diabatic heating. If this is concentrated around the equator in a limited area then convective quasi-equilibrium leads to the vertical structure, and a Gill-like pattern inevitably emerges in the horizontal. The pattern then converges moisture toward its centre, and the ensuing condensation sustains its structure (and produces convective aggregation). The moisture convergence in such a pattern is in fact slightly to the east of the pattern's centre, and if the release of latent heat is more-or-less coincident with that convergence then eastward propagation is unavoidable.

The mechanism sounds simple because it is simple. However, there are many complications that both make a closed mathematical theory (in the usual sense of that word) impossible, and that also make it difficult for comprehensive models to consistently reproduce the phenomena. Perhaps the most serious of these is the representation of precipitation and release of latent heat. In a shallow water model, or any simplified model, this cannot be parameterized without making rather strong assumptions. Here we have explicitly accepted that limitation and assumed only that the latent heat release is related to moisture convergence, and explored how the results depend on that relationship. In a three-dimensional model convection must also be parameterized unless the model has very high resolution. If the parameterization is too sensitive then convection will be triggered too readily and coherence will be lost. On the other hand, if convection is too hard to trigger then the pattern will not be excitable or self-sustaining. Thus, other physical effects inevitably become important and, for example, cloud radiative cooling has been found to play a critical role in some models, and wind-induced evaporation is essential in others. This does not mean that such effects should be considered as driving the phenomena. Rather, it means they may be important in 
maintaining the instabilities necessary to sustain the convection producing the phenomenon. In this picture the genesis on an MJO event naturally occurs over a warm moist ocean, for here there is an external source of heating that can initiate the convection and the convergence. However, once started the system is self-sustained. The velocity of propagation then depends on two main factors:

1. The strength of the disturbance, for this determines the rate of the moisture convergence that sustains the pattern and limits the propagation speed. Thus, the propagation speed scales advectively; that is, with the speed of the fluid that is created by the pattern and is similar to or that speed. Observations also show that the anomalous fluid velocity generated by the pattern is up to a few meters per second, of the same order as the translation speed.

2. The distance of the center of the condensational heating from the center of the pattern, when the pattern is stationary. This is hard to establish observationally, for the pattern will move except in its early stages. Trustworthy results with a comprehensive threedimensional model may help in investigating this.

We have not tried to present an all-encompassing theory, nor is the mechanism for propagation necessarily different from that appearing in other models - it will be unavoidably present in some. Rather, we have tried to identify and isolate the mechanism and present it in simple form, explainable in words.

\section{Acknowledgments}

I would like to thank Sally Lavender, Alison Stirling and Prince Xavier, all of the UK Met Office, for many conversations regarding both the difficulties and the usefulness of comprehensive three-dimensional models. The work was funded by NERC under the Paracon project, and by the Newton Fund under the Weather and Climate Science for Services Partnership.

\section{APPENDIX}

For reference, the geopotential in the Gill solution is, mostly using the notation of Vallis (2017) and in nondimensional form with velocities scaled by $c$ and lengths by $L_{d}$ :

$$
\phi_{G}=\frac{1}{2}\left[q_{0}(x)+q_{2}(x)\left(y^{2}+1\right)\right] \mathrm{e}^{-y^{2} / 4},
$$

where

$$
q_{0}= \begin{cases}\frac{-A k}{r^{2}+k^{2}}\left(1+\mathrm{e}^{-2 r L}\right) \mathrm{e}^{r(L-x)}, & x>L . \\ \frac{-A}{r^{2}+k^{2}}\left[r \cos k x+k\left(\sin k x+\mathrm{e}^{-r(x+L)}\right)\right], & |x|<L \\ 0 & x<-L,\end{cases}
$$


and

$$
q_{2}= \begin{cases}0, & x>L \\ \frac{A}{(3 r)^{2}+k^{2}}\left[-3 r \cos k x+k\left(\sin k x-\mathrm{e}^{3 r(x-L)}\right)\right], & |x|<L \\ \frac{-A k}{(3 r)^{2}+k^{2}}\left[1+\mathrm{e}^{-6 r L}\right] \mathrm{e}^{3 r(x+L)}, & x<-L .\end{cases}
$$

Here, $A$ is the amplitude of the forcing of the geopotential equation and that has the form $A \cos (k x) \exp \left(-y^{2} / 4\right), k=\pi / 2 L, L$ is the prescribed zonal scale of the forcing (scaled by $L_{d}$ ), and $r$ is a damping rate. See Gill (1980) or Vallis (2017) for more detail. The saturated value of humidity may be evaluated by using (5) in conjunction with (9), and the humidity convergence may then be evaluated using the associated expressions for the velocity field. Analytic expressions may in principle be obtained but still need to be evaluated numerically.

\section{References}

Adames, Á. F. \& Wallace, J. M., 2014. Three-dimensional structure and evolution of the MJO and its relation to the mean flow. J. Atmos. Sci., 71, 6, 2007-2026.

Adames, Á. F. \& Wallace, J. M., 2015. Three-dimensional structure and evolution of the moisture field in the MJO. J. Atmos. Sci., 72, 10, 3733-3754.

Arnold, N. P. \& Randall, D. A., 2015. Global-scale convective aggregation: Implications for the Madden-Julian Oscillation. J. Adv. Model. Earth Sys., 7, 4, 1499-1518.

De Szoeke, S. P., Edson, J. B., Marion, J. R., Fairall, C. W. \& Bariteau, L., 2015. The MJO and air-sea interaction in TOGA COARE and DYNAMO. J. Climate, 28, 2, 597-622.

De Szoeke, S. P. \& Maloney, E. D., 2020. Atmospheric mixed layer convergence from observed MJO sea surface temperature anomalies. J. Climate, 33, 2, 547-558.

DeMott, C. A., Klingaman, N. P. \& Woolnough, S. J., 2015. Atmosphere-ocean coupled processes in the Madden-Julian oscillation. Rev. Geophys., 53, 4, 1099-1154.

Fuchs, Ž. \& Raymond, D. J., 2017. A simple model of intraseasonal oscillations. J. Adv. Model. Earth Sys., 9, 1195-1211.

Gill, A. E., 1980. Some simple solutions for heat induced tropical circulation. Quart. J. Roy. Meteor. Soc., 106, 447-462.

Holloway, C. E., Woolnough, S. J. \& Lister, G. M., 2013. The effects of explicit versus parameterized convection on the MJO in a large-domain high-resolution tropical case study. Part I: Characterization of large-scale organization and propagation. J. Atmos. Sci., 70, 5, 1342-1369.

Hsu, P.-C. \& Li, T., 2012. Role of the boundary layer moisture asymmetry in causing the eastward propagation of the Madden-Julian oscillation. J. Climate, 25, 14, 4914-4931.

Hsu, P.-C., Li, T. \& Murakami, H., 2014. Moisture asymmetry and MJO eastward propagation 
in an aquaplanet general circulation model. J. Climate, 27, 23, 8747-8760.

Jiang, X., Waliser, D. E., Olson, W. S., Tao, W.-K. et al., 2011. Vertical diabatic heating structure of the mjo: Intercomparison between recent reanalyses and trmm estimates. Mon. Wea. Rev., 139, 10, 3208-3223.

Khairoutdinov, M. F. \& Emanuel, K., 2018. Intraseasonal variability in a cloud-permitting near-global equatorial aquaplanet model. J. Atmos. Sci., 75, 12, 4337-4355.

Kiladis, G. N., Straub, K. H. \& Haertel, P. T., 2005. Zonal and vertical structure of the Madden-Julian oscillation. J. Atmos. Sci., 62, 2790-2809.

Lau, W. K.-M. \& Waliser, D. E., 2012. Intraseasonal variability in the atmosphere-ocean climate system. 2nd edn. Springer, 613 pp.

Liu, P., Satoh, M., Wang, B., Fudeyasu, H. et al., 2009. An MJO simulated by the NICAM at 14- and 7-km resolutions. Mon. Wea. Rev., 137, 10, 3254-3268.

Madden, R. A. \& Julian, P. R., 1971. Detection of a 40-50 day oscillation in the zonal wind in the tropical Pacific. J. Atmos. Sci., 28, 702-708.

Madden, R. A. \& Julian, P. R., 1972. Description of global-scale circulation cells in the tropics with a 40-50 day period. J. Atmos. Sci., 29, 1109-1123.

Maloney, E. D., 2009. The moist static energy budget of a composite tropical intraseasonal oscillation in a climate model. J. Climate, 22, 3, 711-729.

Mapes, B. E. \& Bacmeister, J. T., 2012. Diagnosis of tropical biases and the MJO from patterns in the MERRA analysis tendency fields. J. Climate, 25, 18, 6202-6214.

Matsuno, T., 1966. Quasi-geostrophic motions in the equatorial area. J. Meteor. Soc. Jpn., 44, $25-43$.

Nasuno, T., Miura, H., Satoh, M., Noda, A. T. \& Oouchi, K., 2009. Multi-scale organization of convection in a global numerical simulation of the December 2006 MJO event using explicit moist processes. J. Meteor. Soc. Jpn., 87, 2, 335-345.

Penn, J. \& Vallis, G. K., 2017. The thermal phase curve offset on tidally and nontidally locked exoplanets: A shallow water model. Astrophys. J., 842, 2, 101.

Raymond, D. J. \& Fuchs, Ž., 2009. Moisture modes and the Madden-Julian oscillation. J. Climate, 22, 3031-3046.

Sobel, A. \& Maloney, E., 2013. Moisture modes and the eastward propagation of the MJO. J. Atmos. Sci., 70, 187-192.

Sperber, K. R., 2003. Propagation and the vertical structure of the Madden-Julian oscillation. Mon. Wea. Rev., 131, 12, 3018-3037.

Vallis, G. K., 2017. Atmospheric and Oceanic Fluid Dynamics. 2nd edn. Cambridge University Press, 946 pp.

Vallis, G. K. \& Penn, J., 2020. Convective organization and eastward propagating equatorial disturbances in a simple excitable system. Quart. J. Roy. Meteor. Soc., 1-18. doi:10.1002/QJ.3792.

Wang, B., Liu, F. \& Chen, G., 2016. A trio-interaction theory for Madden-Julian oscillation. 
Geosci. Letters, 3, 1, 1-16.

Wheeler, M. \& Kiladis, G. N., 1999. Convectively coupled equatorial waves: Analysis of clouds and temperature in the wavenumber-frequency domain. J. Atmos. Sci., 56, 374-399.

Wheeler, M. C. \& Hendon, H. H., 2004. An all-season real-time multivariate mjo index: Development of an index for monitoring and prediction. Monthly weather review, 132, 8, 1917-1932.

Yano, J.-I., McWilliams, J. C. \& Moncrieff, M. W.and Emanuel, K. A., 1995. Hierarchical tropical cloud systems in an analog shallow-water model. J. Atmos. Sci., 52, 1723-1742.

Zhang, C., 2005. Madden-Julian oscillation. Rev. Geophys., 43, 1-36.

Zhang, C. \& McPhaden, M. J., 2000. Intraseasonal surface cooling in the equatorial western Pacific. J. Climate, 13, 13, 2261-2276.

The End. 